\title{
Hand Geometry Identification System using Machine Vision
}

\author{
Gaurav Bansal ${ }^{1}$, Jyoti Saxena ${ }^{2}$ \\ M.tech Research Scholar, Department of Electronics and Communication Engineering, \\ GZS- PTU Campus Bathinda, Punjab, India ${ }^{1}$ \\ Head of Department, ECE Department of Electronics and Communication Engineering, \\ GZS- PTU Campus Bathinda, Punjab, India ${ }^{2}$
}

\begin{abstract}
Authentication based on biometrics is being applied to control physical access to high-security facilities. Recently, with the recent rapid growth of information system technologies, applications for accessing databases or business workflow systems have begun to use biometrics such as hand geometry, palm, iris, fingerprint etc. As the integrity of data is important, We have to guarantee the integrity of the registered biometric data. This paper presents an identification-verification biometric system based on the combination of hand geometry features. The system is designed in NI Vision Assistant 2009. Such approach increased both detection and recognition efficiency of our palm and finger print biometrics system. The proposed system is reliable and user friendly as it is developed in Vision Assistant software. The availability of data log files in Vision Assistant and LabVIEW makes it one of the most useful component as a database.
\end{abstract}

Keywords: Biometrics, palm data base, image pre-processing, NI Vision Assistant 2009, NI LabVIEW 2009.

\section{INTRODUCTION}

The rapid development in the use of information technology and the great concern of security require reliable and automatic personal recognition. Traditional automatic personal recognition schemes can be divided into two categories: Token-based, such as a physical key, an ID card, smart card and knowledge-based, such as an ATM card and a passport. However, these approaches have constriction. In the token-based approach, the "token" can be easily stolen or lost. In the knowledgebased approach, to some extent, the "knowledge" can be guessed, forgotten or shared.[1] These approaches are not sufficiently reliable to satisfy the security requirements because they lack the capability to differentiate between an authentic person and fraud who illegally acquires the access. Biometric recognition, or biometrics, refers to the automatic recognition of a person based on his/her anatomical (e.g., hand geometry, face recognition or iris) or behavioral (e.g., signature) characteristics or traits. This method of recognition offers several advantages over traditional methods involving ID cards (tokens) or PIN numbers (passwords) for various reasons: (i) the person to be recognized is required to be physically present at the point-of-recognition; (ii) recognition based on biometric techniques obviates the need to remember a password or carry a token.[2]

In the 21 st century, unimodal biometrics such as face, iris, voice etc. are widely used for identity verification. But most of these systems suffer from various drawbacks such as the inability to tolerate deformed data due to noise, distorted signal by environmental noise or inconsistency of an individual's physical look and pattern due to long time. Therefore, there is a hand geometry biometrics system which is capable to solve some of these limitations by combining information from more than one biometrics traits. A combination of fingerprint and palm print in a multi modal system will be useful in high security applications. The benefit of combining these features lies in the fact that while taking the data for hand geometry, the data for fingerprints and palm prints can be collected simultaneously. The quality of fingerprint and palm print images is first enhanced using a series of pre-processing techniques. There will be no extra problem to the user while the accuracy of the system may be greatly increased due to the addition of several more features. For instance, an identification/verification system may use fingerprints for identification and use hand geometry for verification. It is easy to conceptualize a sensing system which can simultaneously capture both fingerprints and palm print.

The applicability of a particular hand geometry biometric technique very much depends on the application area. Hand recognition has been available for over twenty years. To achieve personal authentication, a system may measure either physical characteristics of the fingers or the palm. These include length, width, thickness and surface area of the hand. One interesting characteristic is that some systems require a small biometric sample (a few bytes). Hand geometry has gained acceptance in a range of applications. It can repeatedly be found in physical access control in commercial and residential applications, in/out time, attendance systems and in general personal authentication applications. We are used to fingerprints but infrequently think of an entire hand as an individual identifier. This method relies on devices that measure the length, angles and pattern prints of individual hands. To enroll a person in a database, two snapshots of the hand are taken and the average of resulting feature vectors is 
computed and stored. Hand Geometry is employed at $C$ locations like the Colombian legislatures, San Francisco International Airport, day care centers, a sperm bank, welfare agencies, hospitals, and immigration facilities. The only limitation is for people with severe arthritis who cannot spread their hands on the reader.

Hand geometry identification is considered to be the best choice for most applications from network security systems to compact devices, due to its accuracy, speed, reliability, nonintrusive interfaces, cost effectiveness and ease of acquisition. Hand geometry recognition needs a fine Hand image to create and match the templates. But the quality of the acquired hand geometry images is not always good. Therefore it becomes necessary to preprocess the acquired images in order to enhance their quality. So, the main objective of this paper is to develop an efficient Hand geometry pre-processing algorithm using NI Vision Assistant and to validate the identity of a user using pattern matching.[3]

This paper is organized in four sections: In section II, Hand enhancement using various NI vision steps has been discussed, in section III, Result and Discussions are described and section IV gives a conclusion and the future direction.

\section{PALM ENHANCEMENT}

Hand geometry image enhancement algorithm receives an input palm and finger print images then applies the image enhancement steps on the input image of data base of hand and finally at the output gives the enhanced image.[6] The image enhancement algorithm developed is explained step by step in the following sections:

\section{A. Image acquisition}

The first step is to acquire the image by using vision assistant. In Vision Assistant the Image Browser contains all the images currently loaded. An image can be selected from the browser for processing as shown in Figure1.

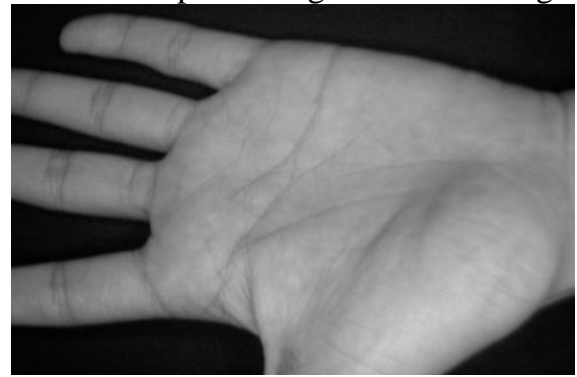

Figure 1. Original Image

\section{B. $\quad$ Extract Color Planes from an Image:}

The grayscale images require less storage space. Therefore, it is beneficial to convert the colored images into the grayscale images. The pattern matching function operates easily upon the grayscale images. Here the hand image data base is in already grey scale so, there is no need to convert. Otherwise we can simply apply the color plane extraction. The number of planes in an image corresponds to the number of arrays of pixels that compose the image. [7]
Image Mask:

Create an image mask from an ROI (region of interest) selected on the image. Mask Options create outside the ROI \& sets all pixels outside the selected region to 0 . Create inside the ROI sets all pixels inside the selected region to 0 .

Extract Masked Region Casts the resulting image to the bounding rectangle of the ROI. We isolate parts of an image for processing by using image mask. An image mask is an 8-bit binary image that is smaller than the inspection images. The image mask contains pixels which determine whether corresponding pixels in the inspection image are processed or not. The required portion of palm image is mask for processing in Figure2.

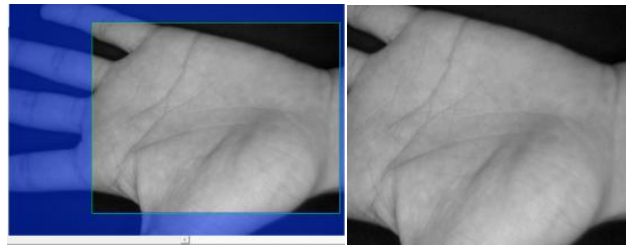

Figure 2. Mask the image

\section{Histogram}

The histogram indicates the quantitative distribution of the pixels of an image per gray-level value. This is a fundamental image analysis tool which also describes the distribution of pixels intensities in an image. The histogram is used to count the total number of pixels in each grayscale value and graph the results. A histogram provides a general description of the appearance of an image and helps identify various components such as background, objects and noise. The histogram is the function $H$ defined on the grayscale range $[0, \ldots, k, \ldots$, $255]$ such that the number of pixels equal to the gray-level value $k$ is $H(k)=n k$ where $n k$ is the number of pixels in an image with a gray-level value equal to $k$, and $\Sigma n k$ from $k$ $=0$ to 255 is the total number of pixels in an image. [3] The histogram setup of masking image is shown as in Figure 3

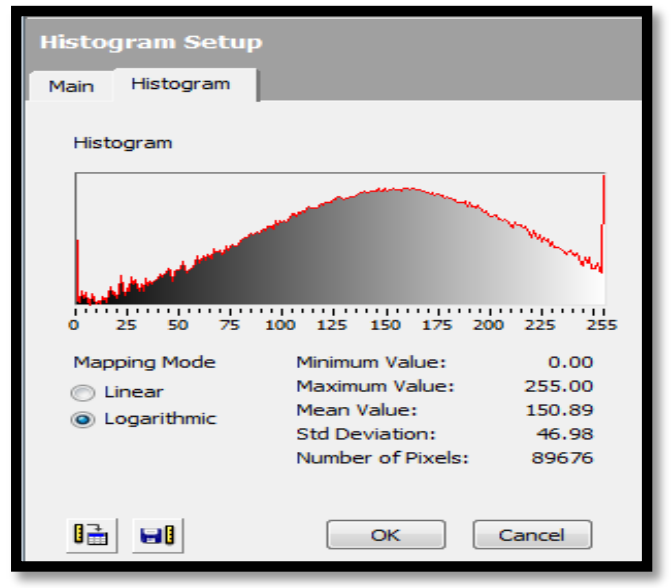

Figure 3. histogram window

$E$ Lookup Table

Grey scale palette groups have several image functions that analyze grayscale images. One of them is lookup 
table. Use this function to improve the contrast and brightness. This consists of image source as equalize, reverse, square, square root, power x etc. Square function is used in this script to improve contrast and brightness.

\section{F. $\quad$ Filters}

These are used to prepare an image for processing so that the information which is needed can be extracted from the image. Filters are used to flat, grind, change and eliminate noise from images. Most of these filters apply a kernel across the image.

To perform filtering in Vision Assistant, we execute the following steps:

a. Choose filters in the Gray scale label.

b. Choose the convolution highlight filter as it highlights the lines of an image showing the sharp palm pattern.

c. Choose the kernel size $3 \times 3$ and press $\mathrm{OK}$.

d. After applying the convolution-custom filter on masked image, the output image is shown as in Figure 4.

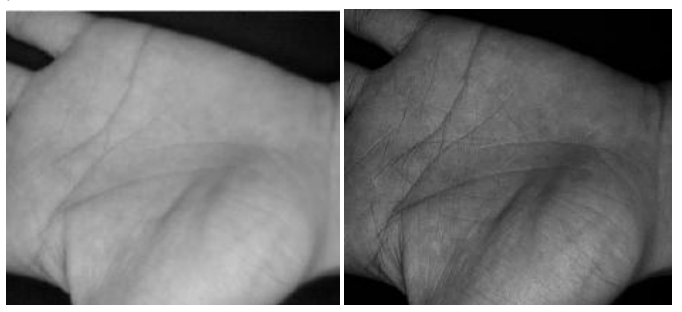

Figure 4. histogram equivalent filter

\section{G. $\quad$ Pattern matching}

The Pattern Matching function works only with 8-bit images. We select an ROI to create templates of different sizes. By default the centre of the template is used as the focal point of the template. We can change the location of the focal point to any position in the template. Modify the focal point of the template by lagging the red indicator in the template image or adjust the Match Offset values. Modify Number of matches to find minimum Score and Sub pixel Accuracy as necessary. The template with pattern matching window is shown in Figure 5.
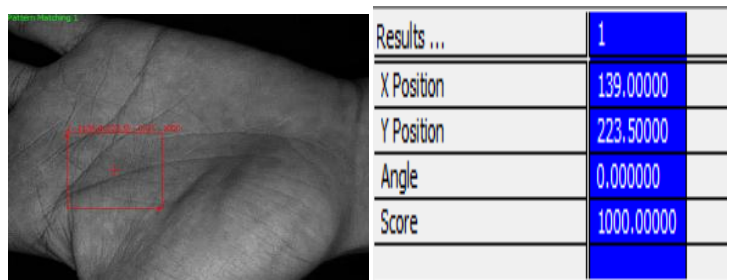

Figure 5. Template with pattern matching

\section{H. Implementing Vision Assistant Script}

Figure 6 corresponds to the script as formed in NI vision assistant where image acquire and pre-processing steps are performed to identify palm pattern.[8]

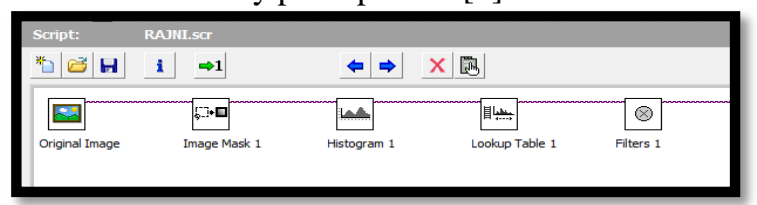

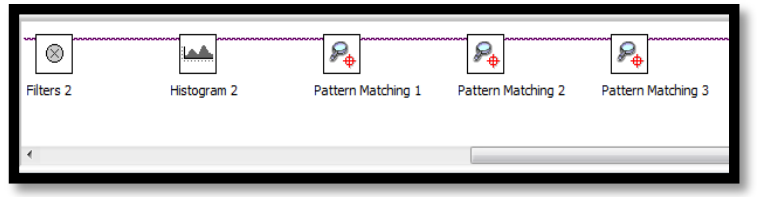

Figure 6. Hand geometry consist palm print as well as finger Scripts in NI Vision Assistant

\section{PERFORMANCE MATRICES}

The experiments are performed on a set of data \& two performance parameters i.e. FRR \& FAR has been calculated to find out the accuracy of the system. The FRR is the frequency that an authorized person is discarded. FRR [14] [15] is generally thought of as a comfort criteria, because a false rejection is most of all annoying. Due to the statistical nature of the false rejection rate, a large number of verification attempts have to be undertaken to get statistical reliable results. FAR is frequency that an unauthorized person is accepted by the system. These two parameters should be as minimum as possible for the better accuracy of system.

\section{RESULT AND DISCUSSION}

In this paper the Hand geometry identification has been designed on image processing -based algorithm. The proposed algorithm has been developed using NI Vision Assistant 2009 and Lab VIEW software. Hand geometry images enhancement algorithm receives an input hand images then applies the image enhancement steps on the input image of data base of hand and finally at the output gives the enhanced image. The values for the False Rejection rate \& False Acceptance Rate for the different template sizes at various thresholds has calculated. In determining the FRR \& FAR, only Palm print from successfully enrolled users are considered. In this paper experiments are conducted by taking templates of three different sizes which are $25 \times 25,35 \times 35,50 \times 50$ as shown in Table1, $2 \& 3$. So, it is concluded that as resolution of the image with the template size is changed, the matching score increases and a perfect match is obtained.

Table1. Overall \%FRR of the system for palm and finger print

\begin{tabular}{|c|c|c|c|c|c|c|}
\hline Threshold & $\begin{array}{c}25 \times 25 \\
(\text { Palm })\end{array}$ & $\begin{array}{c}25 \times 25 \\
(\text { finger })\end{array}$ & $\begin{array}{c}35 \times 35 \\
(\text { Palm })\end{array}$ & $\begin{array}{c}35 \times 35 \\
(\text { finger })\end{array}$ & $\begin{array}{c}50 \times 50 \\
(\text { Palm })\end{array}$ & $\begin{array}{c}50 \times 50 \\
(\text { finger })\end{array}$ \\
\hline 200 & $0 \%$ & $0 \%$ & $0 \%$ & $0 \%$ & $0 \%$ & $0 \%$ \\
\hline 250 & $0 \%$ & $0 \%$ & $0 \%$ & $0 \%$ & $0 \%$ & $0 \%$ \\
\hline 300 & $0 \%$ & $0 \%$ & $0 \%$ & $0 \%$ & $0 \%$ & $0 \%$ \\
\hline 350 & $0 \%$ & $0 \%$ & $1 \%$ & $0 \%$ & $0 \%$ & $6 \%$ \\
\hline 400 & $0 \%$ & $0 \%$ & $2 \%$ & $2 \%$ & $0 \%$ & $16 \%$ \\
\hline 450 & $0 \%$ & $0 \%$ & $5 \%$ & $4 \%$ & $22 \%$ & $30 \%$ \\
\hline 500 & $0 \%$ & $0 \%$ & $23 \%$ & $12 \%$ & $30 \%$ & $44 \%$ \\
\hline 550 & $2 \%$ & $11 \%$ & $44 \%$ & $14 \%$ & $40 \%$ & $52 \%$ \\
\hline 600 & $3 \%$ & $22 \%$ & $61 \%$ & $35 \%$ & $53 \%$ & $56 \%$ \\
\hline 650 & $10 \%$ & $26 \%$ & $68 \%$ & $49 \%$ & $53 \%$ & $64 \%$ \\
\hline 700 & $16 \%$ & $34 \%$ & $72 \%$ & $57 \%$ & $56 \%$ & $64 \%$ \\
\hline 750 & $30 \%$ & $46 \%$ & $75 \%$ & $67 \%$ & $62 \%$ & $66 \%$ \\
\hline 800 & $36 \%$ & $64 \%$ & $82 \%$ & $74 \%$ & $67 \%$ & $72 \%$ \\
\hline 850 & $51 \%$ & $64 \%$ & $89 \%$ & $92 \%$ & $76 \%$ & $72 \%$ \\
\hline 900 & $61 \%$ & $79 \%$ & $92 \%$ & $94 \%$ & $81 \%$ & $79 \%$ \\
\hline
\end{tabular}


INTERNATIONAL JOURNAL OF INNOVATIVE RESEARCH IN ELECTRICAL, ELECTRONICS, INSTRUMENTATION AND CONTROL ENGINEERING Vol. 2, Issue 10, October 2014

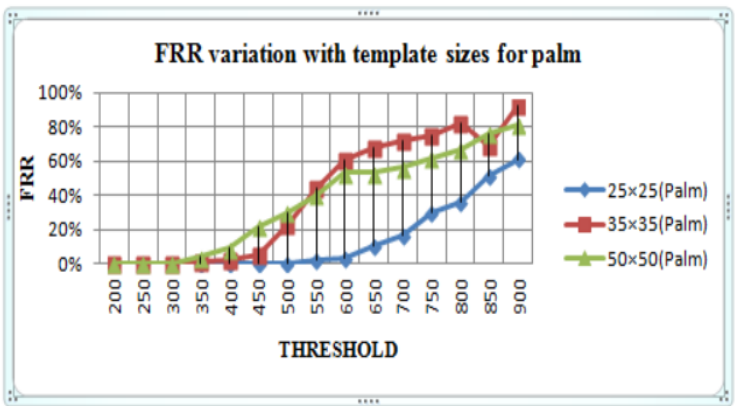

Figure $7 \%$ FRR for learning images, size $25 \times 25,35 \times 35$ and $50 \times 50$ pixels, at various thresholds for palm print

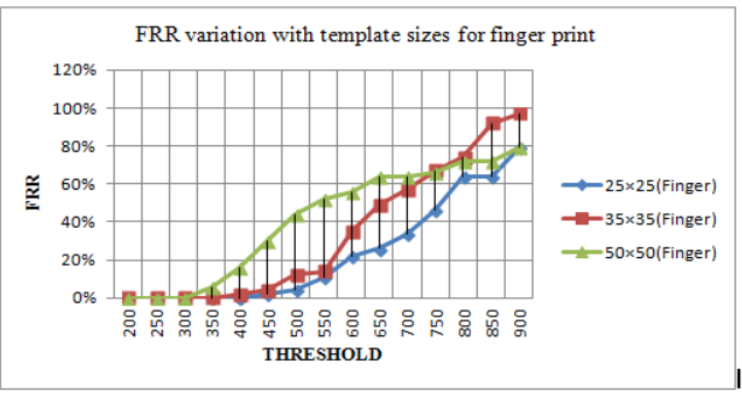

Figure $8 \%$ FRR for learning images, size $25 \times 25,35 \times 35$ and $50 \times 50$ pixels, at various thresholds for finger print

Table $2 \%$ FAR for learning images, size 50×50 pixels, at various thresholds

\begin{tabular}{|c|c|c|c|c|c|c|}
\hline Threshold & $\begin{array}{c}25 \times 25 \\
(\text { Palm })\end{array}$ & $\begin{array}{c}25 \times 25 \\
\text { (finger) }\end{array}$ & $\begin{array}{c}35 \times 35 \\
(\text { Palm })\end{array}$ & $\begin{array}{c}35 \times 35 \\
(\text { finger })\end{array}$ & $\begin{array}{c}50 \times 50 \\
(\text { Palm })\end{array}$ & $\begin{array}{c}50 \times 50 \\
\text { (finger) }\end{array}$ \\
\hline 200 & $100 \%$ & $100 \%$ & $47 \%$ & $68 \%$ & $23 \%$ & $22 \%$ \\
\hline 250 & $100 \%$ & $100 \%$ & $22 \%$ & $47 \%$ & $14 \%$ & $10 \%$ \\
\hline 300 & $100 \%$ & $100 \%$ & $0 \%$ & $23 \%$ & $7 \%$ & $0 \%$ \\
\hline 350 & $100 \%$ & $100 \%$ & $0 \%$ & $14 \%$ & $0 \%$ & $0 \%$ \\
\hline 400 & $100 \%$ & $99 \%$ & $0 \%$ & $7 \%$ & $0 \%$ & $0 \%$ \\
\hline 450 & $98 \%$ & $47 \%$ & $0 \%$ & $0 \%$ & $0 \%$ & $0 \%$ \\
\hline 500 & $95 \%$ & $35 \%$ & $0 \%$ & $0 \%$ & $0 \%$ & $0 \%$ \\
\hline 550 & $79 \%$ & $17 \%$ & $0 \%$ & $0 \%$ & $0 \%$ & $0 \%$ \\
\hline 600 & $69 \%$ & $0 \%$ & $0 \%$ & $0 \%$ & $0 \%$ & $0 \%$ \\
\hline 650 & $48 \%$ & $0 \%$ & $0 \%$ & $0 \%$ & $0 \%$ & $0 \%$ \\
\hline 700 & $35 \%$ & $0 \%$ & $0 \%$ & $0 \%$ & $0 \%$ & $0 \%$ \\
\hline 750 & $17 \%$ & $0 \%$ & $0 \%$ & $0 \%$ & $0 \%$ & $0 \%$ \\
\hline 800 & $11 \%$ & $0 \%$ & $0 \%$ & $0 \%$ & $0 \%$ & $0 \%$ \\
\hline 850 & $5 \%$ & $0 \%$ & $0 \%$ & $0 \%$ & $0 \%$ & $0 \%$ \\
\hline 900 & $0 \%$ & $0 \%$ & $0 \%$ & $0 \%$ & $0 \%$ & $0 \%$ \\
\hline
\end{tabular}

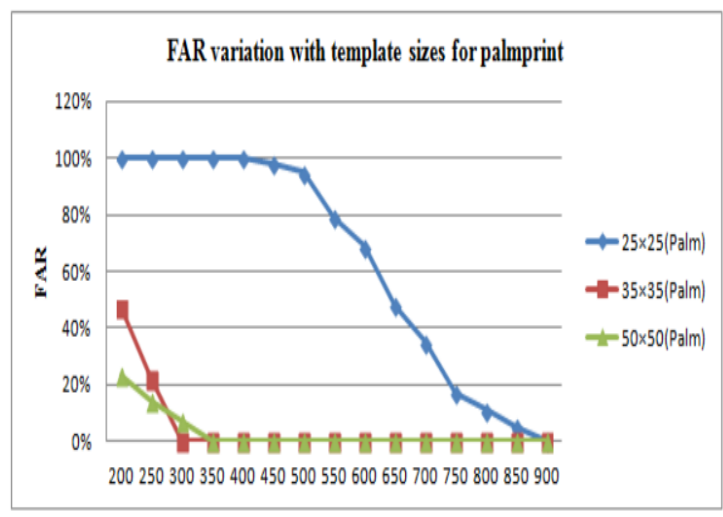

Figure9 \%FAR for learning images, size $25 \times 25,35 \times 35$ and 50×50pixels, at various thresholds for palm print

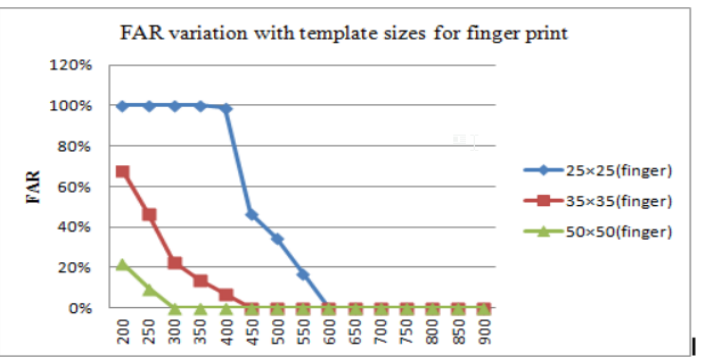

Figure10 \%FAR for learning images, size $25 \times 25,35 \times 35$ and 50×50pixels, at various thresholds for palm print

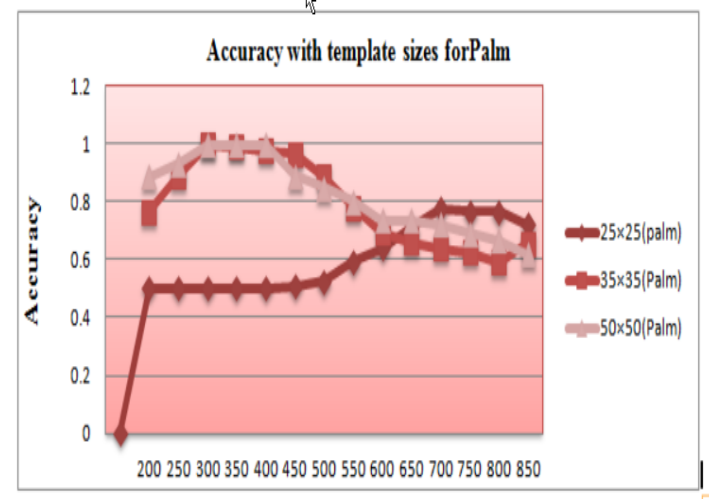

Figure11 Accuracy for learning images, size $25 \times 25$, $35 \times 35$ and $50 \times 50$ pixels, at various thresholds for palm print

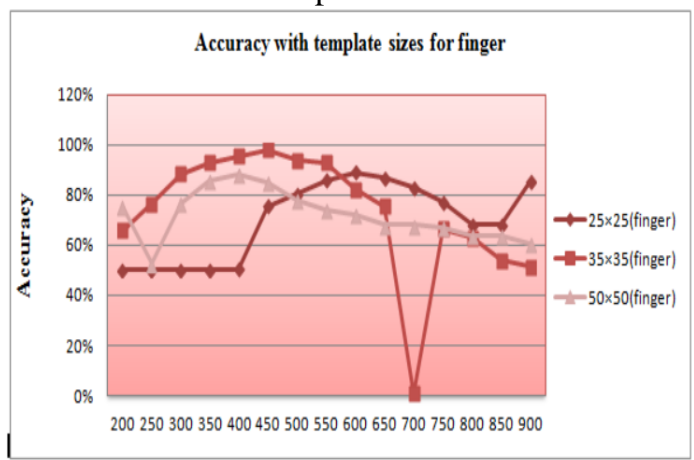

Figure12 Accuracy for learning images, size $25 \times 25$, $35 \times 35$ and $50 \times 50$ pixels, at various thresholds for fingerprint

Table $3 \%$ Accuracy for both palm \& finger at various thresholds

\begin{tabular}{|c|c|c|c|c|c|c|}
\hline Threshold & \multicolumn{5}{|c|}{ Different Template Size for Palm and Finger } \\
\hline & $\begin{array}{c}25 \times 25 \\
\text { (Palm) }\end{array}$ & $\begin{array}{c}25 \times 25 \\
\text { (Finger) }\end{array}$ & $\begin{array}{c}35 \times 35 \\
\text { (Palm) }\end{array}$ & $\begin{array}{c}35 \times 35 \\
\text { (Finger) }\end{array}$ & $\begin{array}{c}50 \times 50 \\
\text { (Palm) }\end{array}$ & $\begin{array}{c}50 \times 50 \\
\text { (Finger) }\end{array}$ \\
\hline 200 & $50 \%$ & $50 \%$ & $76.5 \%$ & $66 \%$ & $88.5 \%$ & $75.5 \%$ \\
\hline 250 & $50 \%$ & $50 \%$ & $89 \%$ & $76.5 \%$ & $93 \%$ & $52.5 \%$ \\
\hline 300 & $50 \%$ & $50 \%$ & $99.5 \%$ & $88.5 \%$ & $99.8 \%$ & $76.5 \%$ \\
\hline 350 & $50 \%$ & $50 \%$ & $99 \%$ & $93 \%$ & $99.8 \%$ & $86 \%$ \\
\hline 400 & $50 \%$ & $50.5 \%$ & $97.5 \%$ & $95.5 \%$ & $97.8 \%$ & $88 \%$ \\
\hline 450 & $51 \%$ & $75.5 \%$ & $96.5 \%$ & $98 \%$ & $89 \%$ & $85 \%$ \\
\hline 500 & $52.5 \%$ & $80.5 \%$ & $88.5 \%$ & $94 \%$ & $85 \%$ & $78 \%$ \\
\hline 550 & $59.5 \%$ & $86 \%$ & $78 \%$ & $93 \%$ & $80 \%$ & $74 \%$ \\
\hline 600 & $64 \%$ & $89 \%$ & $69.5 \%$ & $82.5 \%$ & $73.5 \%$ & $72 \%$ \\
\hline 650 & $71 \%$ & $87 \%$ & $66 \%$ & $75.5 \%$ & $73.5 \%$ & $68 \%$ \\
\hline 700 & $77.5 \%$ & $83 \%$ & $64 \%$ & $71.5 \%$ & $72 \%$ & $68 \%$ \\
\hline 750 & $76.5 \%$ & $77 \%$ & $62.5 \%$ & $66.5 \%$ & $69 \%$ & $67 \%$ \\
\hline 800 & $76.5 \%$ & $68 \%$ & $59 \%$ & $63 \%$ & $66.5 \%$ & $64 \%$ \\
\hline 850 & $72 \%$ & $68 \%$ & $65.5 \%$ & $54 \%$ & $62 \%$ & $64 \%$ \\
\hline 900 & $69.5 \%$ & $85.5 \%$ & $54 \%$ & $51.5 \%$ & $59.5 \%$ & $60.5 \%$ \\
\hline
\end{tabular}




\section{CONCLUSION AND FUTURE DIRECTION}

Accuracy of system has increased significantly at threshold value range of 300-400 for the template size of $50 * 50$. Also, more work can be done on the geometry matching as well as on principle lines, wrinkles \& ridges on the hand. This system has been tested with offline database, it is required that for the real time identification system, the database should be online.

\section{REFERENCES}

[1] Juan José Fuertes, Carlos M. Travieso, Miguel A. Ferrer, Jesús B. Alonso, "Intra Modal Biometric System using Hand Geometry and Palmprint Texture", in Proceedings 44th Annual IEEE International Carnahan Conference on Security Technology, ISBN: 978-1-42447400-4, pp.318-322, USA, 5-8 October 2010.

[2] Ajay Kumar and David Zhang, "Personal recognition using handshape and texture," IEEE Trans. Image Process., Vol. 15, No. 8, pp. 2454-2461, Aug. 2006.

[3] NI Vision Assistant tutorial manual 2009.

[4] Aythami Morales, Ester González, Miguel A. Ferrer, "On the feasibility of interoperable schemes in hand biometrics", accepted at Sensors, special Issue on Hand-Based Biometrics Sensors and Systems, January 2012.

[5] J. Travis and J. Kring, LabVIEW for Everyone: Graphical Programming Made Easy and Fun, 3rd Edition Prentice Hall, July 2006.

[6] National Instruments, "Definition of Machine Vision", Retrieved from www.ni.com/vision.

[7] Miguel A. Ferrer and Aythami Morales, "Hand-Shape Biometrics combining the visible and Short Wave InfraRed Bands", IEEE Transactions on Information Forensics and Security, ISSN: 15566013, Vol. 6, No. 4, pp. 1305-1314, December 2011.

[8] Aythami Morales, Miguel A. Ferrer, Ajay Kumar, "Toward Contactless Palmprint Authentication", IET Computer Vision: Special Issue on Future Trend in Biometric Processing, ISSN: 1751-9632, Vol. 5, No. 6, pp. 407-416, 2011.

[9] Palm database-http://biometrics.idealtest.org/'"

[10] Y. Zhang, D. Sun and Z. Qiu, "Hand-based single sample biometrics recognition", In Proceedings of International conference on Neural Computer \& Application, pp.1835-1844, 2012.

[11] S. Parashar, A. Vardhan, C. Patvardhan and P. K. Kalra, "Design and Implementation of a Robust Palm Biometrics Recognition and Verification System", 6th Indian Conference on Computer Vision, Graphics \& Image Processing , pp. 543-550, E-ISBN 978-0-76953476-3, 2008.

[12] A.K. Jain, A. Ross and S. Pankanti, "A Prototype Hand Geometrybased Verification System", 2nd International Conference on Audio- and Video-based Biometric Person Authentication (AVBPA), Washington, 1999

[13] S. Parashar, A. Vardhan and P.K. Kalra, "Design and Implementation of a Robust Palm Biometrics Recognition and Verification System", Sixth IEEE Indian Conference on Computer Vision Graphics \& Image Processing, pp. 543 - 550,ISBN 978-07695-3476-3, Dec. 2008.

[14] G. Lu, D. Zhang and K. Wang "Palmprint recognition using Eigen palms features", Elsevier Science, Vol. 24, No. 9-10, pp. $1463-$ 1467,June 2003.

[15] Y.L. Ma, F. Pollick, and W. Terry Hewitt, "Using B-spline curves for hand recognition". International Conference of Pattern Recognition, Vol.03, pp. 251-260, 2004.

[16] L. L. Fong1 and W. C. Seng, "A Comparison Study on Hand Recognition Approaches", International Conference of Soft Computing and Pattern Recognition, IEEE Computer Society, 2009.

[17] R. Sahawneh, A. Ibrahim and A. Zabian, "Authentication Method Using Hand Images for Access Control systems", International Arab Journal of e-Technology, Vol. 1, No. 4, June 2010. 